\title{
Software defined elastic optical networking in temporal, spectral, and spatial domains
}

\author{
S. J. Ben Yoo • Lei Liu • Roberto Proietti • \\ Ryan P. Scott
}

Received: 30 March 2014 / Accepted: 17 May 2014 / Published online: 3 July 2014

(C) The Author(s) 2014. This article is published with open access at Springerlink.com

\begin{abstract}
This paper discusses architecture, protocol, technologies, systems, and networking testbed for softwaredefined elastic optical networking in temporal, spectral, and spatial domains. By exploiting the progress in elastic optical networking (EON) in temporal and spectral domains utilizing dynamic optical arbitrary waveform generation and measurement technologies, and by extending the EON concept into the spatial domain through the new orbital angular momentum-based spatial division multiplexing, we realize EON exploiting elasticity in temporal, spectral, and spatial domains (3D-EON). Routing, spectral, spatial mode, and modulation format assignment with fragmentation awareness as well as hitless defragmentation for high capacity, high quality of service, and resource-efficient networking will be pursued. OpenFlow-based 3D-EON testbed at UC Davis includes optical supervisory channel with optical performance monitoring for software-defined networking with an adaptive observe-analyze-act cycle.
\end{abstract}

Keywords Elastic optical networking - Flexible grid networking · Orbital angular momentum · Arbitrary waveform measurement - Arbitrary waveform generation

\author{
S. J. Ben Yoo $(\bowtie) \cdot$ L. Liu $\cdot$ R. Proietti $\cdot$ R. P. Scott \\ Department of Electrical and Computer Engineering, University \\ of California, Davis, CA 95616, USA \\ e-mail: sbyoo@ucdavis.edu \\ L. Liu \\ e-mail: leiliu@ucdavis.edu \\ R. Proietti \\ e-mail: rproietti@ucdavis.edu \\ R. P. Scott \\ e-mail: rpscott@ucdavis.edu
}

\section{Introduction}

\subsection{Motivation}

The sustained pattern of exponential data traffic growths has completely transformed the landscape of modern telecommunication networks. Initially, the increases in capacity demands were successfully met by deployment of wavelength-division multiplexing (WDM) technology, which offered orders of magnitude increases in communication capacity from $10 \mathrm{~Gb} / \mathrm{s}$ to multiple Tb/s. More recently, rapidly emerging new services and data centers are driving the peak link capacity demands beyond $10 \mathrm{~Tb} / \mathrm{s}$, hence, recent optical networking advances have addressed coherent optical communications with high spectral efficiency beyond $1-10 \mathrm{~b} / \mathrm{s}-\mathrm{Hz}$ employing advanced modulation formats to achieve link capacity as high as $100 \mathrm{~Tb} / \mathrm{s}$. However, it is extremely difficult to support such high spectral efficiency and high capacity under dynamically changing traffic conditions especially due to their sensitivity to physical layer impairments such as fiber amplifier noise, chromatic and polarization dispersion, and optical nonlinearity [1] even for moderate $(\sim 500 \mathrm{~km})$ transmission distances. Hence, capacity beyond $100 \mathrm{~Tb} / \mathrm{s}$ must seek a new dimension other than temporal and spectral domains for growth. The spatial domain is the final unexplored frontier of capacity growth. In the past, space-division multiplexing (SDM) has constantly been an alternative to time-division multiplexing (TDM) and WDM, but now, we must look into a new coherent optical communication method exploiting temporal, spectral, and spatial domains. Figure 1 [2] shows steady increases in spectral efficiency reported for high-capacity transmission systems, which reached $100 \mathrm{~Tb} / \mathrm{s}$ capacity and $10 \mathrm{~b} / \mathrm{s} / \mathrm{Hz}$ spectral efficiency on a single-mode fiber [3], and branching off to SDM for $>100 \mathrm{~Tb} / \mathrm{s}$ capacity toward $10 \mathrm{~Pb} / \mathrm{s}$. SDM also helps to 
(a)

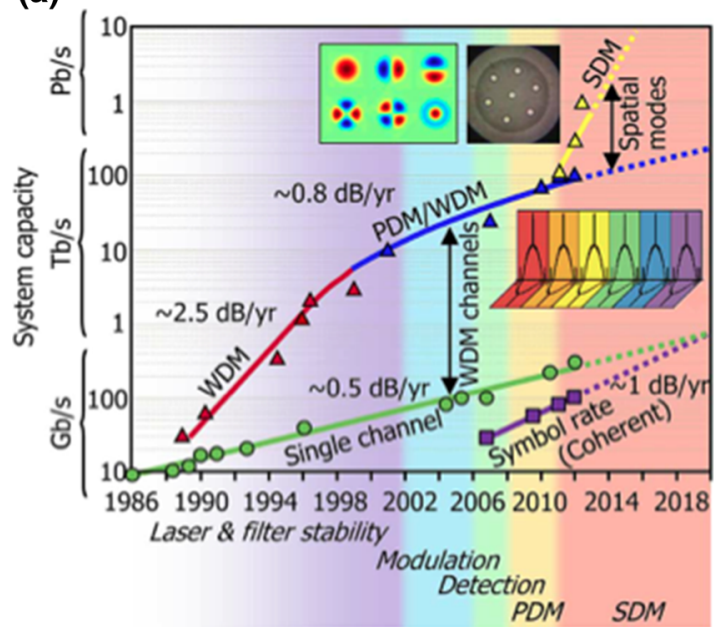

(b)

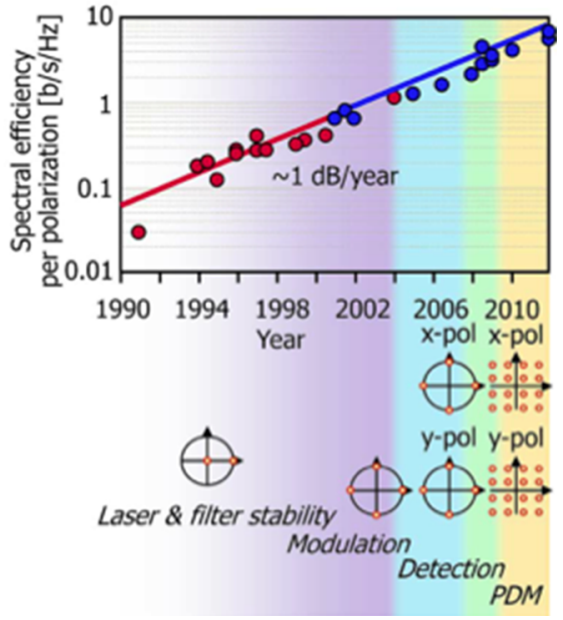

Fig. 1 a Evolution of experimentally achieved single-channel bit rates (single-carrier, single-polarization, electronically multiplexed; green circles), symbol rates in digital coherent detection (purple squares), and aggregate per-fiber capacities (triangles) using wavelength-division multiplexing (WDM; red), polarization-division multiplexing (PDM;

overcome the nonlinear capacity limit on a single-mode fiber [4].

In addition to the increased and flexible capacity use, future optical networks emphasize manageability and controllability of the networks. The networking community has so far adopted two types of network control frameworks: centralized and distributed control systems. Telecom networks have traditionally used centralized control planes for more accurate accountability and security while datacom networks have emerged from simple networking with rich end-system functionality based on the decentralized multiadministrative structure. As the datacom and telecom networks started to converge, telecom networks have considered the distributed Generalized Multi-Protocol Label Switching (GMPLS) architecture and its associated protocols [5] for IP-over-optical networking. While GMPLS provided flexibility for edge devices and functionalities, signaling schemes are still very complicated and it lacked a direct control and management of network resource. More recently, softwaredefined networking (SDN)-based on a centralized controller (e.g., NOX [6]) emerged. While the main concept of SDN is surprisingly similar to reconfigurable Multi-wavelength Optical NETworking pursued by the MONET consortium involving telecom companies in 1993-1999 [7] in separating the control plane from the data plane, and in offering hardware-independent software-defined programmable network elements, the OpenFlow-based SDN benefits from open source codes, in contrast to the fact that GMPLS is usually deployed in closed systems. SDN requires virtualization of networking resources, and it can benefit greatly from elasticity in networking resources in temporal, spectral, and spatial domains. blue), and space-division multiplexing (SDM; yellow) [2]. b Evolution of experimentally achieved per-polarization spectral efficiencies in single-(red) and dual-polarization (blue) experiments [2] (Color figure online)

This paper investigates software-defined elastic optical networking in temporal, spectral, and spatial domains for future high performance cyberinfrastructures. The remainder of the paper is organized as follows. Section 1.2 summarizes the main concepts and feature of EON. Sections 2.1 and 2.2 introduce the two main enabling technologies used to exploit elasticity in the temporal/spectral and spatial domain, namely optical arbitrary waveform generation and measurement (OAWG-OAWM) and orbital angular momentum (OAM), respectively. Section 2.3 discusses how the two technologies above can be combined to form a 3D-EON communication system. Section 3 discusses the routing, spectral, spatial mode, and modulation format assignment (RSSMA) algorithm, which is an evolution of the RSMA considering the possibility to use the additional space domain for routing. Section 4 discusses the defragmentation problem augmented by the new spatial domain. Section 5 discusses some aspects of the SDN control plane design for the proposed 3D-EON. Section 6 discusses how the supervisory channel and performance monitoring technique demonstrated in [8] for a 2D EON scenario can be applied also in the new 3D elastic optical networking. Section 7 summarizes our 2D/3D-EON testbed. Finally, Sect. 8 concludes the paper.

\subsection{Elastic optical networking (EON)}

Recently, a new optical networking technology called elastic optical networking (EON) has been proposed $[9,10]$. As Fig. 2 illustrates, EONs utilize flexible (or elastic) spectral bandwidths for each data link without using fixed wavelength grids. The flexibility in spectrum allocation brings many appealing features to network operations. Current net- 


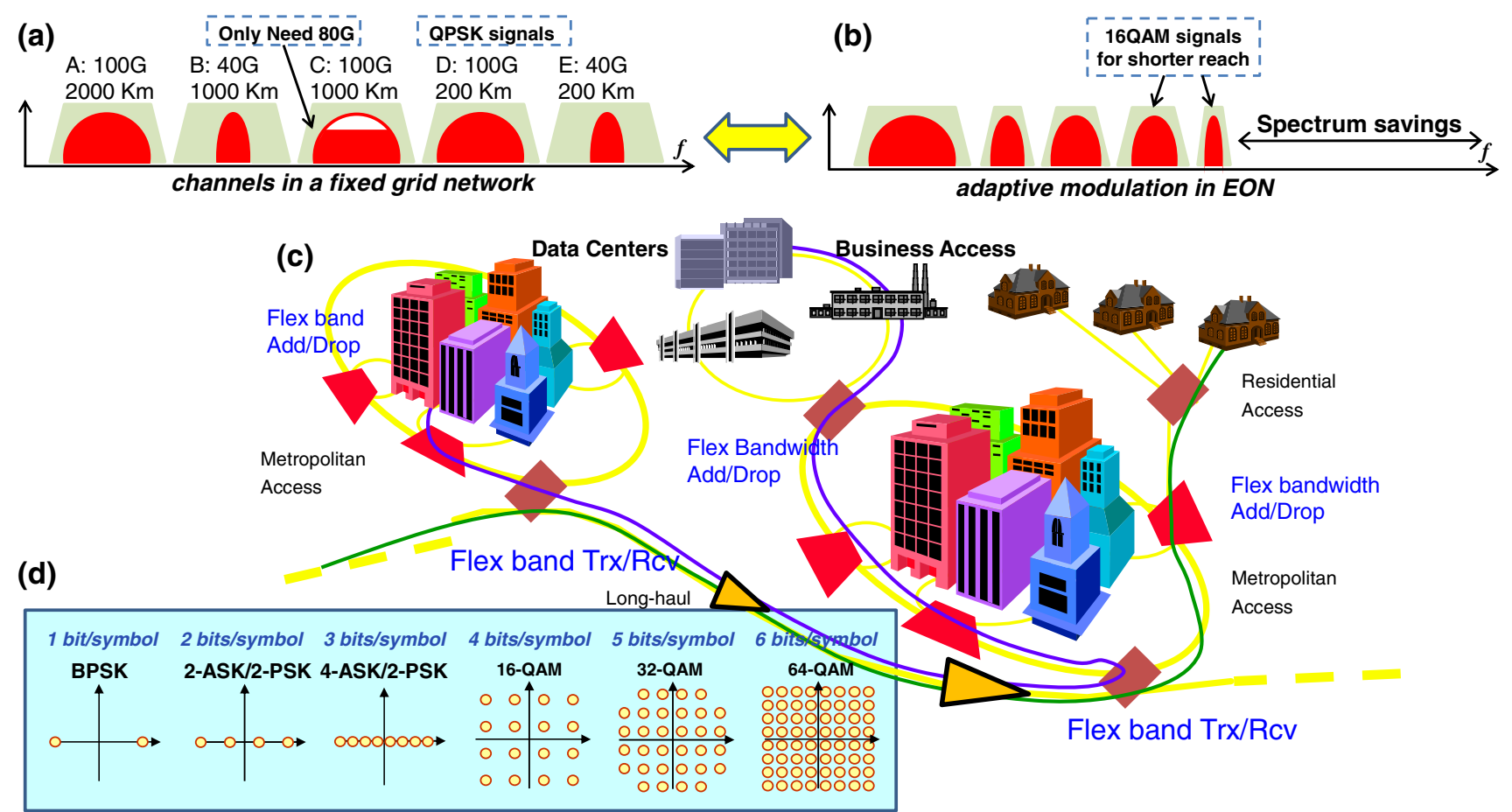

Fig. 2 a WDM networks with five channels on a fixed grid and with fixed modulation format (e.g., QPSK). b using adaptive modulation optimized for the required bitrate and reach, the same demand can be satisfied with considerable spectrum savings [9]; c overview of metropol- itan and access telecom network with flexible bandwidth TRXs and flexible add/drop multiplexer; $\mathbf{d}$ different modulation formats that can be used in EON works are designed for the worst case impairments in transmission performance and the assigned spectrum is overprovisioned. In contrast, the flexible-bandwidth networks can operate with the highest spectral efficiency and minimum bandwidth for the given traffic demand. In the case of a link failure in the network, flexible-bandwidth networks are more adaptive and likely to have spare spectrum to allocate the re-routed signal, ensuring a high survivable restoration compared to conventional optical networks. EONs employ coherent optical orthogonal frequency division multiplexing (CO-OFDM) [11,12], coherent optical WDM (CO-WDM) [13], or Nyquist-WDM technologies [14], and adopt various modulation formats depending on the reach. More recently, UC Davis team has demonstrated dynamic optical arbitrary waveform generation (OAWG) and optical arbitrary waveform measurement (OAWM) [15,16]-based EON, which have overcome a number of limitations of CO-OFDM, COWDM, and Nyquist-WDM technologies, providing opportunities for new architectures to be implemented $[9,15]$. EON in temporal and spectral domain promises (a) to achieve high spectral efficiency by eliminating stranded spectrum between the fixed grid bandwidths, (b) to support both subchannel and superchannel traffic, (c) to provide multiple data rate and modulation formats. However, while EON can achieve $1.2 \times \sim 1.6 \times$ improvement in the network capacity, utilization, and availability, it cannot achieve $10 \times \sim 100 \times$ enhance- ments unless the spatial domain is explored. We will investigate EON in the temporal, spectral, and spatial domains.

\section{Key enabling technologies for EON}

\subsection{Dynamic OAWG and OAWM technologies for EON in spectral-temporal domains}

We will use dynamic OAWG for EON transmitters and dynamic OAWM for EON receivers to exploit elasticity in temporal and spectral domains. As Fig. 3a illustrates, dynamic OAWG $[15,16]$ begins with a coherent optical frequency comb (OFC), which is spectrally demultiplexed with narrow passbands placing each comb line at a separate spatial location. A set of in-phase and quadrature-phase modulators (I/Q modulators), each with a bandwidth of $\Delta f_{G}$, apply temporal I/Q modulations to broaden the comb lines to create the spectral slices. Coherently combining the spectral slices using a gapless spectral multiplexer with broad overlapping passbands ensures a continuous bandwidth output waveform [17]. In this manner, arbitrary waveform (in amplitude and phase) across $>$ THz bandwidth can be generated using standard CMOS electronics $(<20 \mathrm{GHz})$. Preliminary experimental results achieved 240, $360 \mathrm{~Gb} / \mathrm{s}$, and $1.2 \mathrm{~Tb} / \mathrm{s}$ data generation with BPSK, QPSK, and 16QAM modulation formats $[18,19]$. 
(a) Dynamic Optical Arbitrary

Waveform Generation

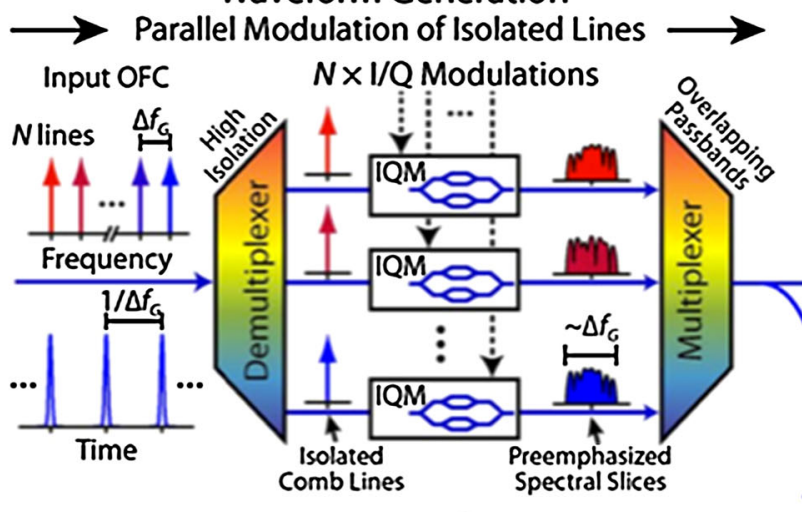

(c) Demultiplexer Transmission

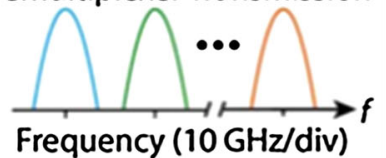

Frequency $(10 \mathrm{GHz} / \mathrm{div})$ (d)

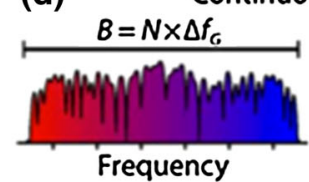

(b)

Optical Arbitrary

Waveform Measurement

Parallel Coherent Detection of Slices $M \times$ Measured I/Q Signals

Reference OFC
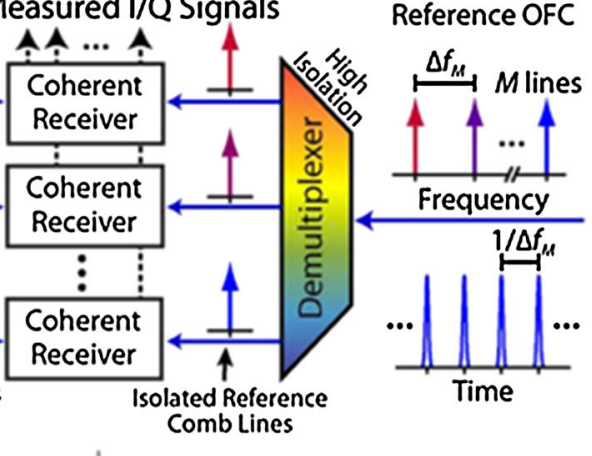

(e) Multiplexer Transmission

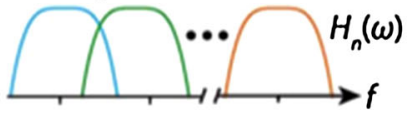

Frequency $(10 \mathrm{GHz} /$ div)
Fig. 3 a Dynamic OAWG transmitter and b OAWM receiver principle. Spectral slice a OAWG and $\mathbf{b}$ OAWM techniques for bandwidth scalable generation and detection of arbitrary modulation format data

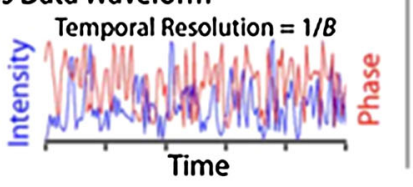


In this transmission system, $\Delta f_{G}$ can be different from $\Delta f_{M}$ as long as the total measurement bandwidth $\left(M \times \Delta f_{M}\right)$ is greater than the generated waveform's bandwidth $\left(N \times \Delta f_{G}\right)$. The use of spectral slices enables independent optimization at the transmitter and receiver for the exact bandwidth of available electronics, and also allows utilization of the transmitters and receivers across heterogeneous network domains.

The OAWG and OAWM can all-optically pre-compensate or post-compensate for chromatic dispersion [18,19], and they can also incorporate all-optical passband pre-emphasis or post-compensation filtering to equalize the non-uniform RF response of the modulators and detectors to ensure high-fidelity waveform generation after the multiplexer [17]. In addition, all-optical pre-compensation and postcompensation can shape the waveform to maintain the lowest peak-to-average power ratio (PAPR) across the transmission link to suppress nonlinear impairments. While recent flexible bandwidth networking and EON research activities have extensively used CO-OFDM, CO-WDM, or Nyquist-WDM technologies, the dynamic OAWG/OAWM technology has the following key advantages over the aforementioned counterparts for EON to be pursued here [9].

1. The dynamic OAWG/OAWM technology can generate and detect signals of any modulation format across the flexible bandwidth. Hence, OAWG/OAWM is backward compatible with other technologies.

2. While CO-OFDM and CO-WDM technologies can generate flexible bandwidth signals of multiple modulation formats, each group of the band must be parked on fixed frequency grids. On the other hand, the dynamic OAWG/OAWM technology can utilize any frequency spacing. Since CO-OFDM and CO-WDM rely on frequency comb with rigid frequency grid (due to the fixed channel spacing of the MUX and DEMUX used in the TX), the spectral efficiency decreases if the line rate per comb line decreases. Co-OFDM and Co-WDM systems can use a flexible grid only when wavelength-selective switches with flexgrid technology are used as MUX and DEMUX in the TRX architecture. On the other hand, dynamic OAWG/OAWM technique allows generation of arbitrary signal channels on arbitrary spacing as desired even when using a fixed frequency comb and MUX and DEMUX with fixed channel spacing.

3. While CO-OFDM, CO-WDM, and Nyquist-WDM technologies can generate coherent optical spikes and very high PAPR [23], the OAWG/OAWM technology can shape the total waveform to suppress PAPR and nonlinear impairments. In fact, since the DSP in OAWG can shape the total waveform at its output, rather than the waveform of each single subcarrier, this can potentially be used to control the PAPR evolution along the link by shaping the waveform in such a way that minimizes the nonlinear effects and maximizes the quality of the received signals.

\subsection{Spatial domain enabling technology: orbital angular momentum (OAM)}

An additional degree of freedom in the spatial domain can exploit orbital angular momentum (OAM) [24], so that together with dynamic OAWG and OAWM, it will enable 3D EON in the temporal, spectral, and spatial domains. Among many spatial domain methods, OAM [24] is attractive because it supports well-defined orthogonality and cylindrical symmetry. As Fig. 5a illustrates, the light beam carrying orbital angular momentum exhibits an azimuthal phase variation of $\varphi(r, \phi)=\exp (i \ell \phi)$, where $\phi$ is the azimuthal angle and $\ell$, a positive or negative integer, is the charge number of the OAM state. The OAM states of differing charge numbers $(\ell)$ are orthogonal to each other and the handedness of this helical phase front is determined by the sign of the charge number $\ell$. For a nonzero charge number, the OAM beam leads to a phase singularity on the beam axis, and the amplitude along the beam axis vanishes. Compared to a spin mode (i.e., polarization), the light beam can in principle support an infinite number of OAM states at the same time (subject to the Shannon limitation imposed by the signal to noise ratio). Therefore, OAM has the potential to significantly improve the spectral efficiency or photon efficiency of free-space and fiber optical communications [25]. In addition, the inherent orthogonality of the various OAM states may reduce crosstalk, resulting in reduced reliance on digital signal processing (DSP) compared to cases employing standard multiple-input and multiple-output (MIMO) methods [25].

As illustrated in Fig. 5b, the UC Davis team has demonstrated [26] OAM multiplexing and demultiplexing devices based on photonic integrated circuits (PICs) containing a free propagation region (FPR) employing a Rowland circle. At the Roland circle, the wavefronts acquire a linear phase tilt based on the input waveguide position, thus providing progressive phase evolution in the azimuthal angle as required for the OAM state. The superposition principle of waves applied to Fig. $5 b$ indicates that multiplexing of multiple OAM states is possible, and propagating the waves in reverse will achieve demultiplexing of multiple OAM states. Figure $5 \mathrm{c}$ illustrates a recent realization including a $3 \mathrm{D}$ photonic integrated circuit $[27,28]$ interfacing with the 2D PIC containing the FPR with a Roland circle. The 3D PIC is written by 3D laser inscription $[29,30]$ that works similarly as a 3D printer but uses a tightly focused ultrafast laser beam. The fabricated 3D/2D PIC supports Mux/Demux of up to 15 OAM states, with both TE and TM polarizations and relatively low loss performance at $1.55 \mu \mathrm{m}$. The elasticity in the spatial domain can be achieved by including all or part of the available 15 


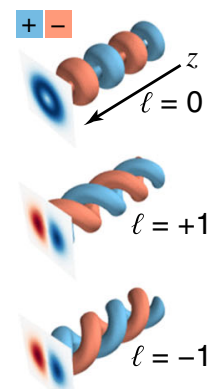

(a)

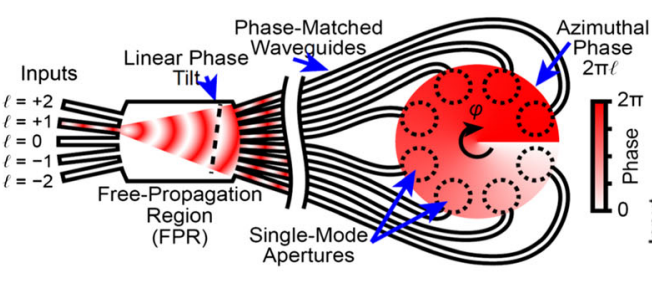

(b) (c)

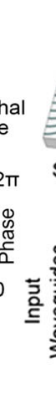

Fig. 5 a 3D illustration of OAM mode propagation for $\ell=0,+1,-1$. b Planar waveguide configuration of OAM multiplexer/demultiplexer which includes the Rowland circle free-propagation region (FPR) and the phase-matched waveguides interfacing antenna apertures placed on a perimeter of the circle. Thus, the OAM multiplexer converts the linear phase front tilt to the azimuthal varying phase. Input OAM states at $\ell=+2,+1,0,-1,-2$ will each get projected to an array of phase-

OAM states in the network, similarly to the way modulation formats are assigned. The compact design and single-mode interface easily connect with other high-speed optical components for future on-chip integration. The recent successful OAM transmission and switching experiments [26,27,31] exploited these 3D/2D PICs. We report below an example of OAM free space transmission exploiting the OAM PIC device described above.

In this experiment, we demonstrate a polarization-diversified free-space OAM link with simultaneous transmission of three OAM states and 14 wavelength channels using two OAM hybrid devices as a OAM mux/demux pair. Each wavelength channel has $10 \mathrm{GBd}$, dual-polarization quadraturephase shift keying (DP-QPSK) data for an aggregate capacity of $1.68 \mathrm{~Tb} / \mathrm{s}$ and spectral efficiency of $9.6 \mathrm{bits} / \mathrm{s} / \mathrm{Hz}$.

Figure 6a shows a conceptual diagram of a photonic integrated circuit (PIC) for OAM state multiplexing/demultiplexing. The free-propagation region (FPR) applies a linear phase tilt on the input light. The phase-matched waveguides sample the output of the FPR and transform the linear phase tilt to azimuthal phase variations at the output apertures. Illuminating multiple inputs simultaneously generates OAM states that spatially overlap at the output apertures, providing true OAM state multiplexing. Alternatively, when operated in reverse, the circular-patterned apertures sample and demultiplex spatially overlapped OAM states. Figure 6b shows a drawing of the 2D-3D hybrid integrated device where the 2D silica PLC couples with a 3D waveguide circuit fabricated by direct laser inscribing in borosilicate glass. Figure $6 \mathrm{c}$ shows the normalized transmission from OAM mux input (A) to OAM demux output (B) for both polarizations. The total loss for OAM state 0 is $19.4 \mathrm{~dB}$ (device TE) and $19.3 \mathrm{~dB}$ (device TM). Figure $6 \mathrm{~d}$ shows the experimental arrangement where a 10 GBd QPSK signal (2 $2^{15}-1$ PRBS) drives all 14 WDM

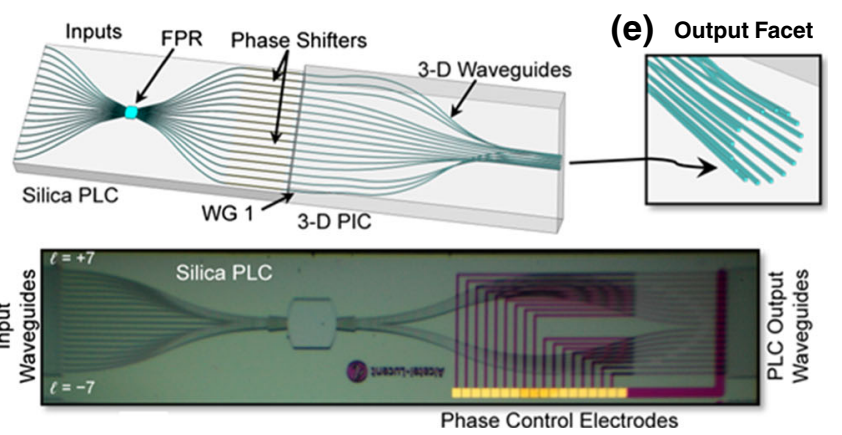

(d)

matched waveguides to excite apertures with azimuthally varying phase dependent on the OAM state number $\ell$. This way, multiple OAM states can be multiplexed spatially (SDM). c Construction of an OAM mux/demux based on a 2D silica planar lightwave circuit (PLC) containing the FPR and phase shifters and 3D waveguides leading to an output facet with apertures along the ring. $\mathbf{d}$ a photograph of the $2 \mathrm{D}$ silica PLC

channels (12.5 GHz spacing). The modulated signal is polarization multiplexed with decorrelation of 500 ns between the two polarization states. After multiplexing up to three OAM states through the first hybrid device, the spatially overlapped beams are transmitted over a $81.8 \mathrm{~cm}$ free space link, demultiplexed by the second hybrid device and sent to the polarization-diversified coherent receiver with offline DSP. The DSP uses an adaptive equalizer, which includes a 17-tap finite impulse response (FIR) filter, power-of-four methods for phase and frequency recovery, and direct-decision-based bit error counting.

The averaged bit error rate (BER) of two polarization states for a multichannel free space transmission link was measured. We used OAM states $-6,-3$ and 0 for the transmission link experiment. The measured crosstalk values at all three OAM states outputs are between -10.5 and -11.6 $\mathrm{dB}$ for TE, between -10.2 and $-14.1 \mathrm{~dB}$ for TM. Figure $7 \mathrm{a}$ shows the measured BER for the following cases: (i) back to back transmission without WDM or OAM states crosstalk (XT); (ii) back to back transmission with all the wavelength channels; (iii) single OAM state and single wavelength channel transmission without crosstalk from other OAM states; (iv) all three OAM states transmission with single wavelength channel; (v) single OAM state with all the wavelength channels but without crosstalk from other OAM state; (vi) all three OAM states transmission with all the wavelength channels. There is $\sim 3 \mathrm{~dB}$ OSNR penalty for OAM state 0 and -3 transmission with OAM states crosstalk. However, the OSNR penalty for OAM state -6 is much higher due to a joint effect of crosstalk and polarization dependent loss (PDL). In the future, the better phase error correction can further reduce the crosstalk. PDL is mainly from the misalignment in the device setup. Figure $7 \mathrm{~b}$ shows that the measured averaged BER values with all three OAM 


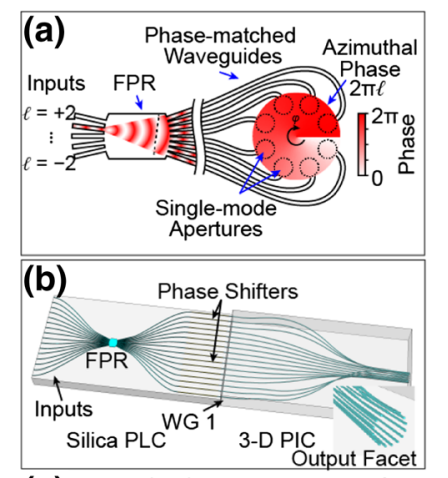

(d) $10 \mathrm{GBd}$ QPSK EAWG

(c) Normalized Transmission Power for
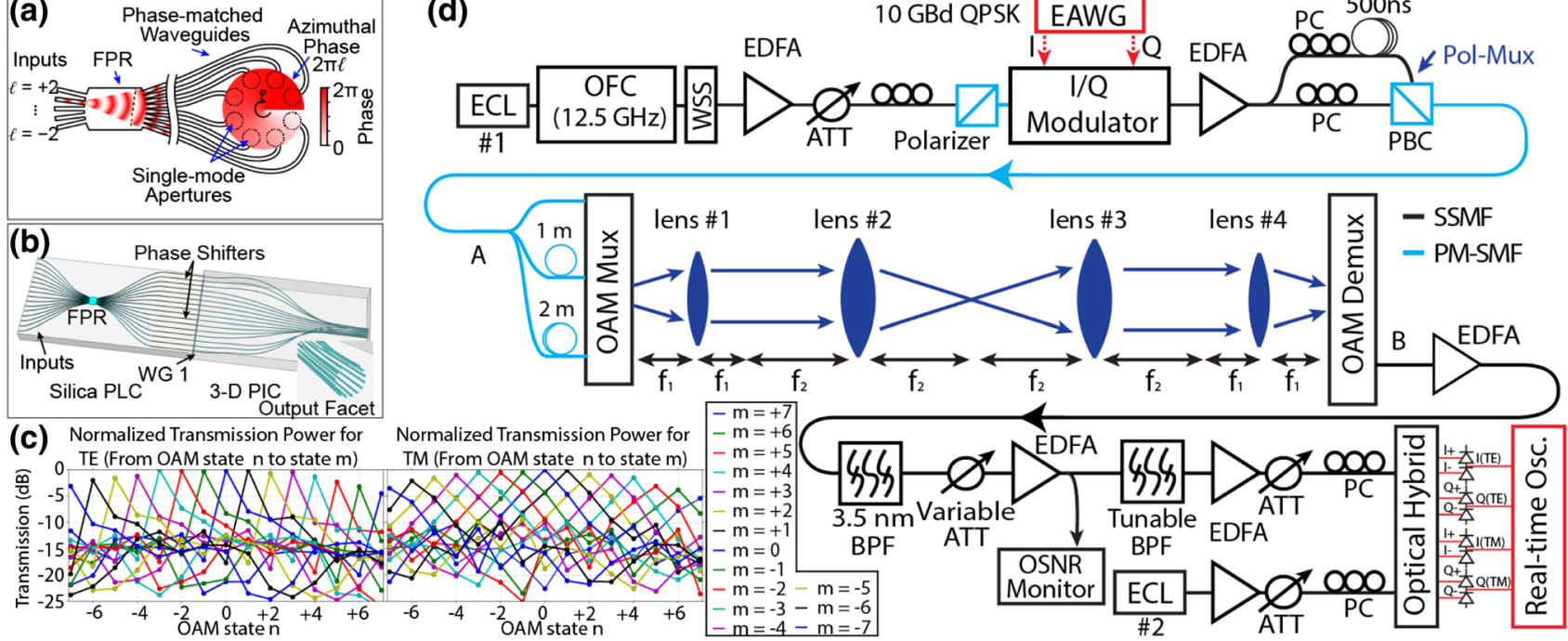

Fig. 6 a Concept of the OAM photonic integrated circuit. b Conceptual view of the 2D-3D hybrid integrated device for OAM mux/demux. c Normalized transmission of the OAM mux/demux pair. d Experimental arrangement of the multichannel OAM polarization-diversified freespace communication link testbed. FPR free-propagation region; $E C L$

external cavity laser; $O F C$ optical frequency comb; WSS wavelengthselective switch; EDFA erbium-doped fiber amplifier; $A T T$ attenuator; $P C$ polarization controller; $P B C$ polarization beam combiner; $B P F$ bandpass filter. $E A W G$ electrical arbitrary waveform generator

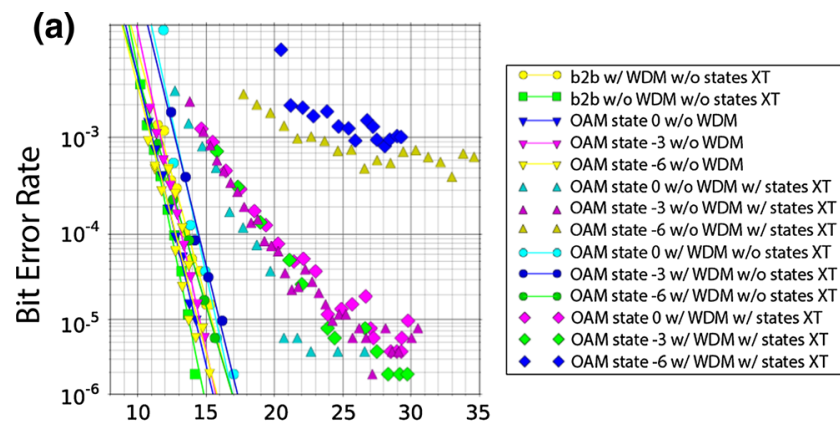

OSNR (dB@0.1nm bandwidth resolution)

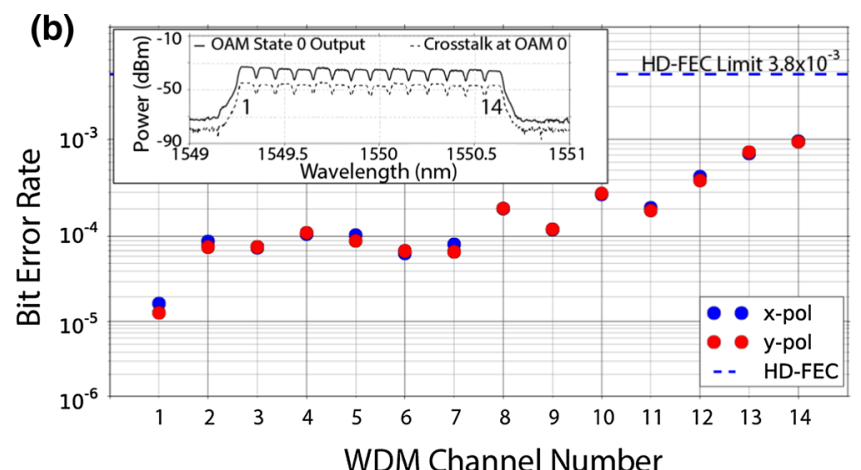

Fig. 7 a BER performance for the multichannel OAM link with $14 \times 10 \mathrm{GBd}$, DP-QPSK WDM channels. b Averaged BER for three OAM states $(0,-3$ and -6$)$ for two received orthogonal polarizations ( $x-$ pol and y-pol not necessarily the same as device TE and TM)

states at all wavelength channels are below HD-FEC limit of $3.8 \times 10-3(\mathrm{BCH}(1020,988)$ super FEC code, $7 \%$ overhead).

\subsection{System architecture for EON in spatial-temporal-spectral domains}

The experimental example shown at the end of the above section is a practical simple example of how temporal and spectral domain can be combined with spatial domain. In fact, multiple WDM-QPSK signals illuminating multiple inputs of the OAM MUX simultaneously generate OAM states that spatially overlap at the output apertures, providing true OAM state multiplexing. At the RX, the same component used in the opposite direction acts as DEMUX to spatially separate the different OAM states.

More in general, as Fig. 8a shows, the 3D EON transmitter in the spatial, temporal, and spectral domains can be constructed by combining multiple parallel temporal and spectral OAWG transmitters feeding signals into the OAM Mux through a unitary matrix. The OAM Mux has been already introduced as a 2D/3D PIC (Fig. 5c), where circular-patterned apertures at the output facet will emit the superposition of OAM states weighted by the coefficients $a_{-m}, a_{-(m-1)}, \ldots a_{m}$. The unitary matrix provides the proper (arbitrary) weighting of $a_{-m}, a_{-(m-1)}, \ldots a_{m}$ contributed by each OAWG transmitter. Similarly, Fig. 8b shows the 3D EON receiver in spatial, temporal, and spectral domains constructed by combining multiple parallel OAWM receivers 

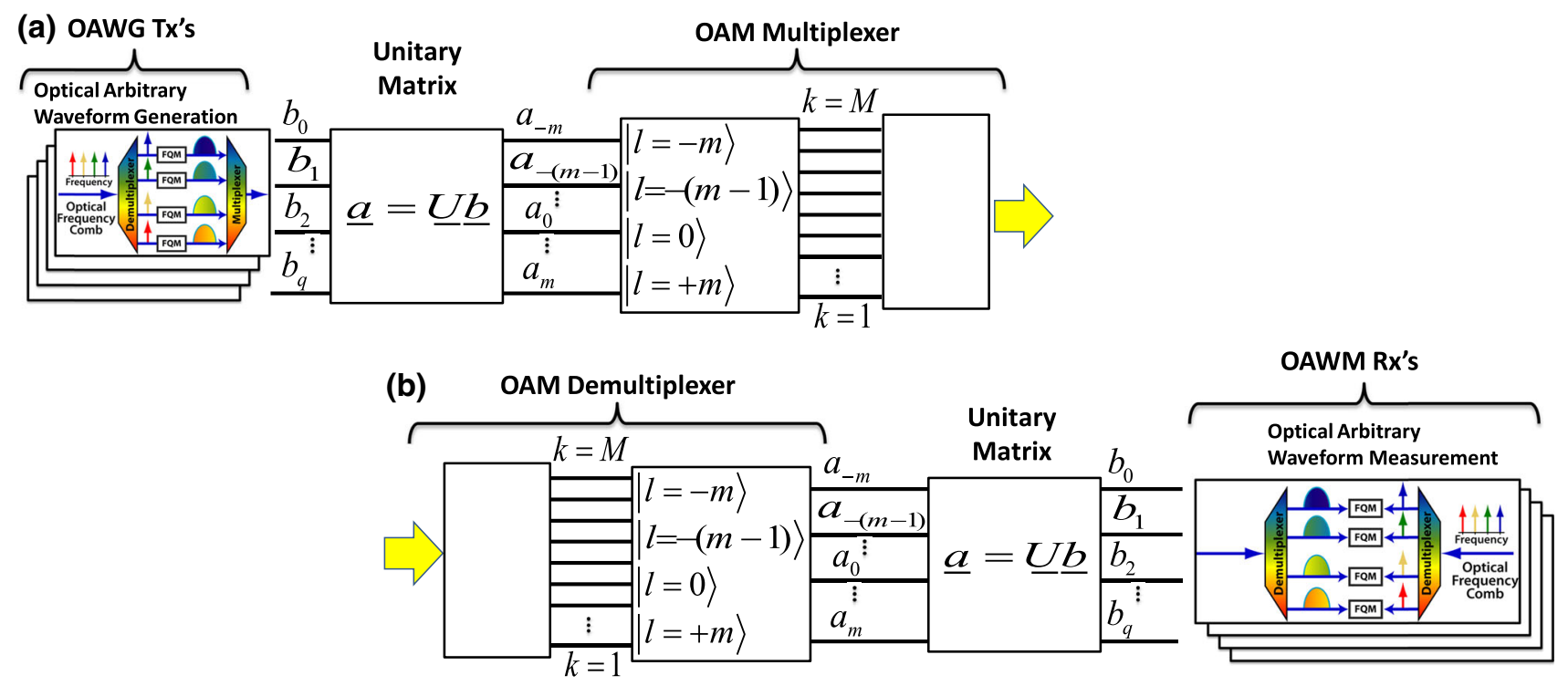

Fig. 8 a 3D EON transmitter in spatial, temporal, and spectral domains constructed by combining multiple parallel OAWG transmitters feeding signals into the OAM Mux through a unitary matrix. The unitary matrix provides the transformation necessary to create a desired (arbitrary) superposition of multiple OAM states for each OAWG Tx. b 3D

receiving signals from the OAM Demux through a unitary matrix. The unitary matrix provides the necessary transformation to map the received (arbitrary) superposition of multiple OAM states for each OAWM Rx.

\section{RSSMA in temporal-spectral-spatial domains}

In standard EONs, we investigated routing, spectral, and modulation format assignment (RSMA) [32-37] along with defragmentation in the spectral domain. In [37], the proposing team investigated fragmentation-aware and alignmentaware RSMA algorithms so that the need for future defragmentation becomes minimal. In the proposed 3D EONs, we add the additional degree of freedom in the spatial mode (OAM states) and investigate routing, spectral, spatial mode, and modulation format assignment (RSSMA) with similar fragmentation awareness and alignment-awareness.

The fragmentation problem in the proposed EONs has three dimensions, namely those of spectral, spatial, and temporal fragmentation. Taking an example network as shown in Fig. 9a, the vertical dashed rectangular indicates the fragmented spectrum resources in link $\mathrm{AB}$, which is the fragmentation in the spectral dimension. On the other hand, Fig. 9b depicts the spatial dimension fragmentation when multiple OAM states are introduced. We should consider the spectral, spatial and temporal dimensions when designing solutions to the fragmentation problem. Also, considering the fact that spectrum misalignment between neighboring links will most probably increase the end-to-end blocking probability,
EON receiver in spatial, temporal, and spectral domains constructed by combining multiple parallel OAWM receivers receiving signals from the OAM Demux through a unitary matrix. The unitary matrix provides the necessary transformation to map the received (arbitrary) superposition of multiple OAM states for each OAWM Rx

as shown in the horizontal dashed rectangular in Fig. 9b, therefore, an optimized RSSMA algorithm should assign a new connection in such a way that it fragments the least number of continuous spectral blocks on candidate links, while it fills up as many misaligned spectral slots as possible on neighboring links, for a given OAM state.

Figure 10 shows a more detailed example of the effect on the spectral fragments before and after a lightpath provisioning process for a given OAM state. Suppose that we have a 6-node, 8-link mesh network and that the spectral resources are distributed as shown in Fig. 10a. For simplicity, in this example, we assume only 12 spectrum slots on each fiber link. When a new request " $A$ to $E$ " arrives with a bandwidth requirement of 1 slot, the routing algorithm first calculates all possible routes, resulting in the 5 shortest paths as shown in Fig. 10b. There are 6 paths from $A$ to $E$ in total, but path $A D B C F E$ is omitted since we consider only " $k$ " $(k=5)$ shortest paths for each source-destination pair. All arrows in Fig. 10b indicate the different possible routes and spectral positions to provision the requested circuit " $A$ to $E$ ". Note that, when a spectral block with more than one slot is available for this one-slot request, the first-fit rule applies and only the slot assignment in the bottom (the lowest spectrum frequency) of the block is taken. In Fig. 5b, one "cut" indicates that the candidate RSSMA solution will break the contiguousness of a spectral block on one of the links on the current path. The "cuts" are considered to be the costs of the candidate solutions, since more "cuts" create more fragments on the candidate links of the routes. For example, the provisioning of the request on path $A B C E$ with slot 10 will "cut" two 


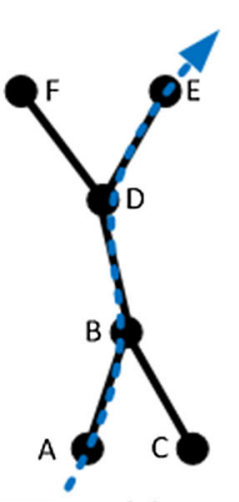

(a)

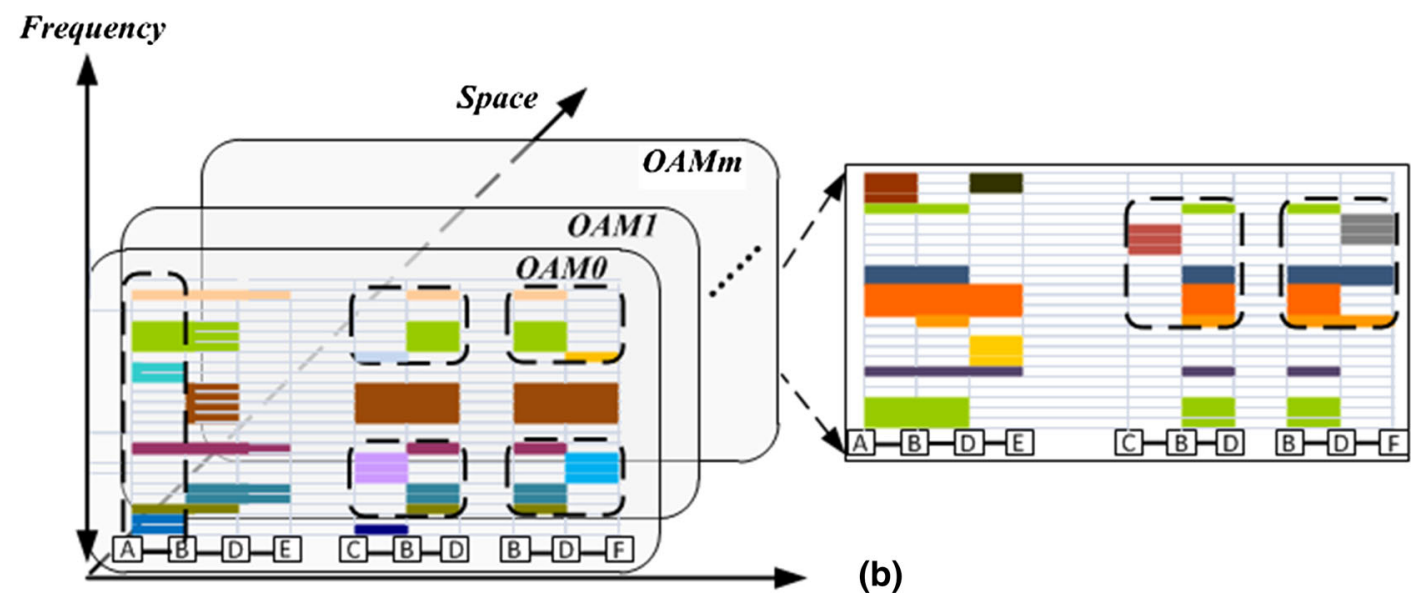

Fig. 9 a An example topology for elastic optical networking; b spectral fragmentation on spectral-spatial-temporal dimensions

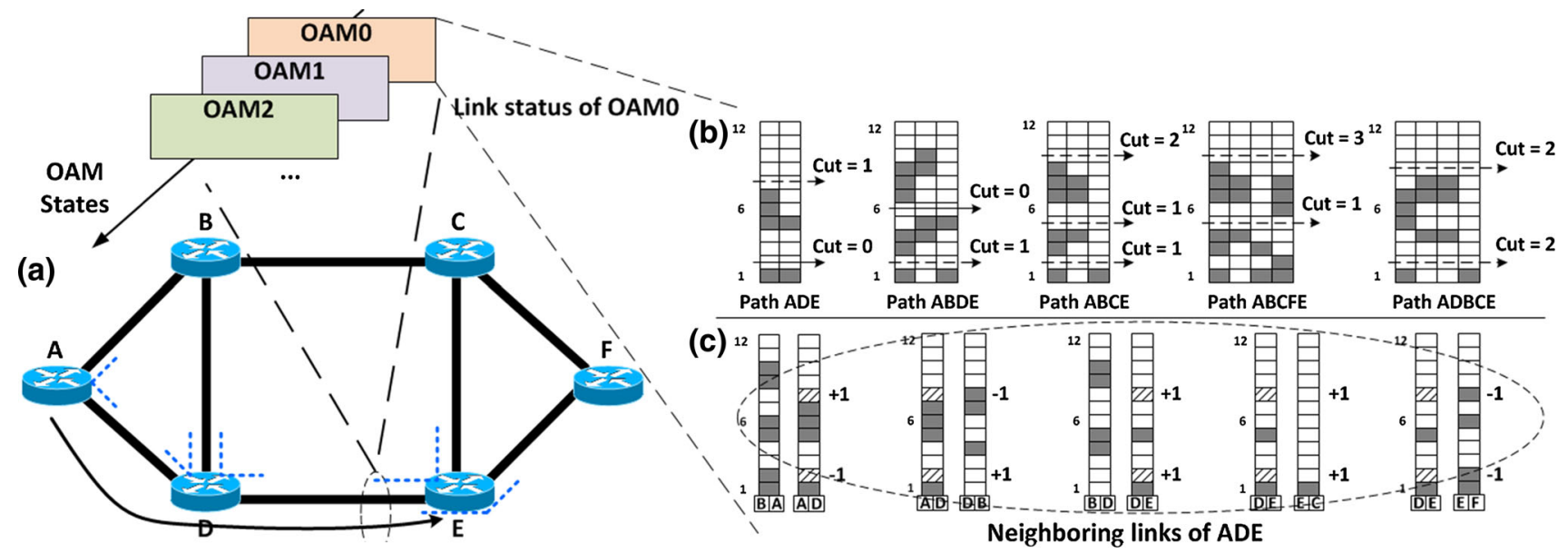

Fig. 10 a Example FISH network and the spectral assignment status on the links, b number of "Cuts" to all the candidate solution to connection request $A-E$, and c misalignment increase if choosing path $A D E$ with slot 8 or slot 2

spectrum blocks on links $B C$ and $C E$, namely the contiguous spectral slots 9-12 on link $B C$ and spectral slots $2-12$ on link $C E$. Likewise, the other provisioning choices "cut" different numbers of spectrum blocks on their corresponding routes. The spectrum blocks become more fragmented as they lose continuousness in the spectral domain. In the example, in Fig. 10b, the provisioning choices with solid arrows on the path $A D E$ and $A B D E$ both give zero "cuts"; therefore, they are the most preferred solutions in terms of spectral fragmentation awareness. On the other hand, the provisioning of a request can also increase the misalignment of the available spectral blocks between the candidate links and their neighboring links. The optimized spectrum assignments are keeping the unused spectrum on neighboring links aligned for future requests. For example, the candidate provisioned on slot 8 (the top slash blocks) will change the alignment of the neighboring links as shown in Fig. 10c. The misalignment for the link pair $B A$ and $A D$ will increase by one since the provisioning on link $A D$ on slot 8 reduces the commonly available spectrum by one slot. Likewise, all the misalignment changes of neighboring link pairs along the candidate path $A D E$ can be calculated. Note that, if the provisioning fills up the originally misaligned spectrum, as shown for link pair $D E$ and $E F$ in Fig. 10c, the misalignment decreases by one. If the requested bandwidth is more than one slot, the "misalignment increase" is counted accumulatively over all slots. The increased misalignment in a network is considered as an additional cost for future light-path provisioning and spectrum defragmentation. Therefore, among all candidate solutions, the algorithm should minimize the misalignment cost as well. For a given OAM state, the proposed fragmentation-aware RSSMA simply gives higher priority to the cost "cuts" and calculates the spectral fragmentation first. It tries to minimize the number of "cuts" on the candidate routes and spectrum slots. If there is more than one solution that achieve the identical minimum number of "cuts", then the algorithm starts to calculate the misalignment increase between the candidate links and their neighboring links. In the end, the algorithm 
provides the optimized RSSMA solution with both minimum "cuts" and minimum increase in misalignment. The shortestpath first-fit rule kicks in if more than one RSSMA solutions are found. If no RSSMA solution can be found for this OAM state, the algorithm will repeat the above procedure and check the feasible solutions for the next OAM state until a RSSMA solution is found.

To show the benefits of the 3D EON and fragmentationaware RSSMA algorithm, we have conducted simulations on sample network topologies to compare the blocking probability of newly proposed fragmentation-aware RSSMA algorithm with our previously proposed $2 \mathrm{D}$ fragmentation-aware RSMA algorithm [37] for traditional EON. Dynamic connection arrival and departure events are simulated on a 14-node NSFNET network and on a 24-node USBN network, respectively. Each spectral slot is set to be $12.5 \mathrm{GHz}$, and each fiber link has 400 slots. In the simulation, each source-destination pair generates connection requests randomly according to a Poisson process. The offered load is controlled by changing the average of the Poisson process in the range over [0.8, 10] arrivals per time unit. The holding time of each connection follows a negative exponential distribution averaging 5 time units. The connection bandwidth is randomly distributed in the range of $L=$ [1-slot, 10-slot]. The number of OAM states is set to 8 for the 3D-EON. Figure 11 compares the blocking probability of the different provisioning algorithms in the 14-node NSFNET and the 24-node USBN network. The results show that fragmentation-aware RSSMA algorithm for 3D EON can greatly reduce the blocking probability compared with our previously proposed $2 \mathrm{D}$ fragmentation-aware RSMA algorithm for traditional EON.

\section{Defragmentation in spatial-spectral domain}

As mentioned above, the fragmentation problem in the 3D EON is not simply related to the spectral domain, but also to the new spatial domain. Therefore, in 3D EONs, it becomes necessary to consider spectral-spatial domain defragmentation. In the standard EON, defragmentation required either wavelength conversion or reassignment of spectrum or routes. Interestingly, in the 3D EON, the OAMstate conversion [38] is far easier than wavelength conversion or time-slot interchange.

The first step in 3D defragmentation is to reconfigure the network so that the fragments can be consolidated into contiguous blocks. While the fragmentation-aware RSSMA should minimize the need for defragmentation, the reconfigurations for defragmentation can be done either periodically or on-demand in the 3D space. Periodical defragmentation usually operates with the goal of confining the spectral usage to one side of the spectrum and requires the entire network to be considered for defragmentation simultaneously.
On the contrary, dynamic on-demand defragmentation can be adaptively tailored to the specific demand of the important and urgent incoming connections. With either approach, the existing connections need to be reconfigured either by changing routes, assigning different spectrum and/or OAM states at the transceivers, or converting wavelength and/or states in the intermediate nodes. It is important to avoid or minimize disruption of live connections. UC Davis team has demonstrated hitless defragmentation [39] in EON by introducing a tunable laser. For 3D EONs, we can also realize hitless defragmentation in the temporal, spectral, and spatial domains by utilizing tunable components.

\section{SDN control plane for 3D EON}

Control plane is a key element to enable intelligent optical networks, especially for the dynamic and cost-efficient provisioning and restoration of end-to-end optical connections. For the 3D EON, two different control planes can be deployed. One is the distributed GMPLS with an optional, dedicated path computation element (PCE). The other one is the centralized SDN architecture, which offers hardwareindependent software-defined network programming capability. The advantages and disadvantages of GMPLS/PCE and SDN, as well as their comparison have been widely discussed in previous studies, such as [40-42]. In this work, we focus on the SDN-based solution, in particular, the OpenFlow-based architecture and its associated protocol. The reason is that OpenFlow-based SDN provides satisfactory flexibility for the operators to control a network given its open interfaces as well as the open nature of its source code, in contrast to the fact that GMPLS is usually deployed in closed systems. In addition, OpenFlow-based SDN requires virtualization of networking resources, and it can benefit greatly from elasticity in networking resources in temporal, spectral, and spatial domains.

Figure 12 shows the OpenFlow-based SDN architecture for the 3D EON. An intelligent OpenFlow-based controller can be deployed to support multi-thread processing and can dynamically perform RSMMA and defragmentation through its PCE module. The PCE can get the network information from the traffic engineering database (TED) for the path computation, and after a successful path computation, the PCE can notify the OpenFlow engine to send the extended OpenFlow messages to the corresponding OpenFlow agents for path provisioning.

To support 3D EON using an OpenFlow-based control plane, all the network elements (NEs) in the 3D EON are required to be extended with the OpenFlow capability. This can be achieved by introducing an OpenFlow agent on top of each NE, as shown in Fig. 12. A centralized OpenFlow controller (e.g., NOX) can communicate with all the Open- 

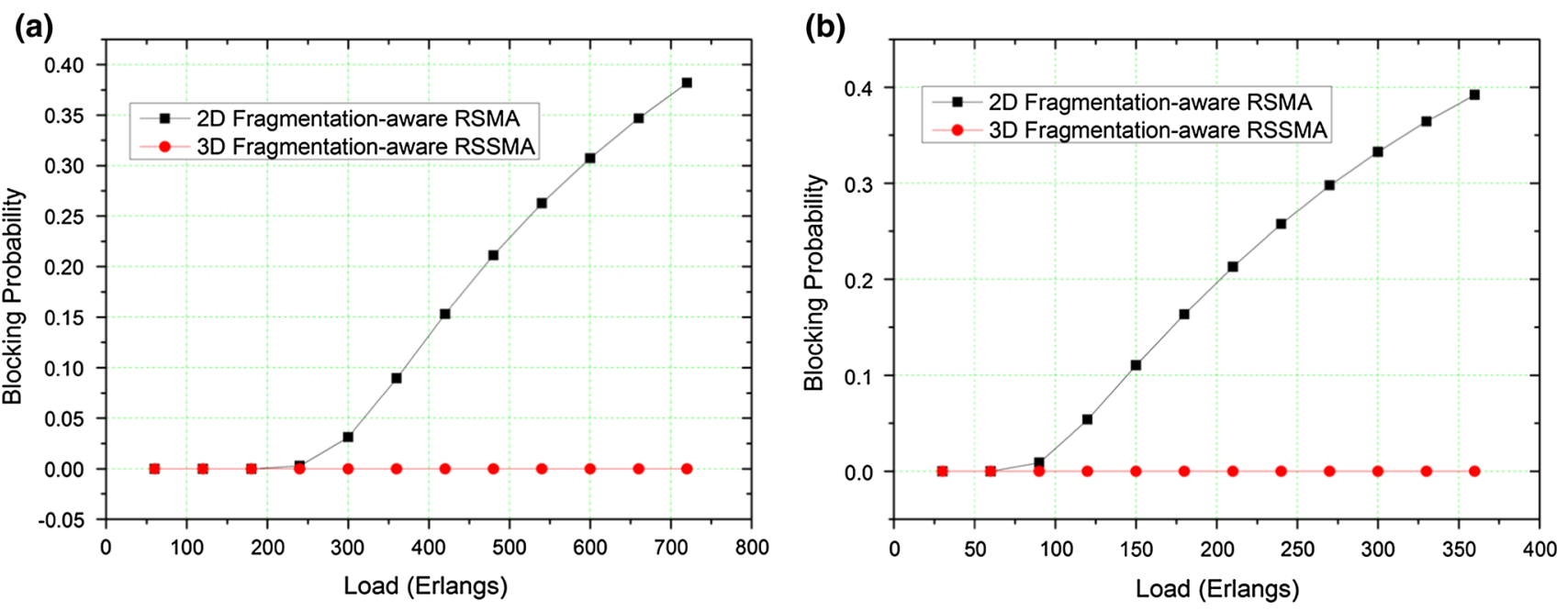

Fig. 11 Blocking probability comparison of different algorithms in the a 14-node NSFNET network, b 28-node USBN network

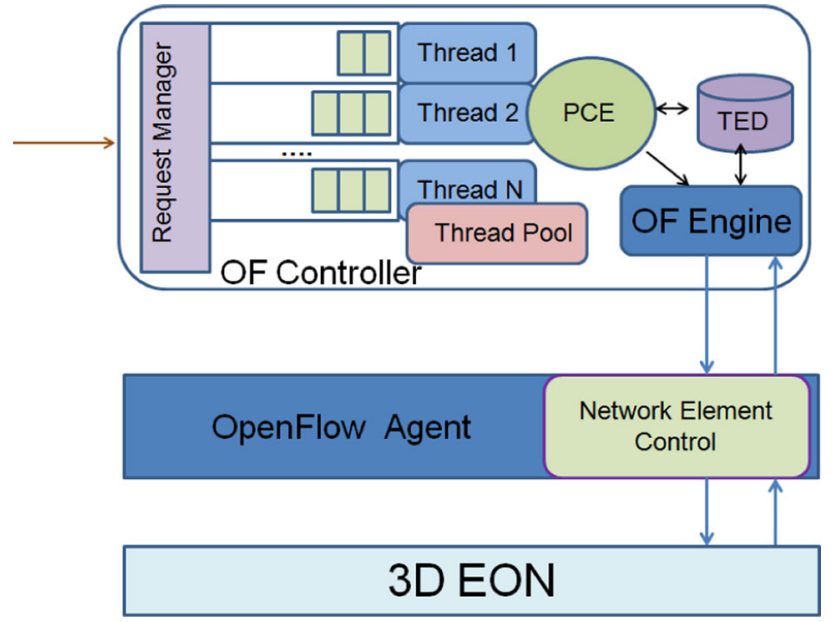

Fig. 12 OpenFlow-based SDN architecture for 3D EON

Flow agents through the extended OpenFlow protocol. The OpenFlow protocol extensions are summarized below:

1. The Feature Reply message is extended to report the new features of a 3D EON (e.g., switching capability, OAM states, available spectrum ranges, etc.) to the NOX controller;

2. The Packet In message is extended to carry the bit rate of each incoming flow

3. The Flow Mod message is extended to carry the RSMMA results from the NOX, including input/output ports, OAM state, central frequency, slot width, and modulation format, etc. for each OpenFlow agent to control the underlying hardware.

\section{Supervisory channel and performance monitoring for 3D EON}

3D EON can benefit from optical supervisory channels that can achieve optical performance monitoring so that pertinent link states can be measured across the entire spectrum and spatial modes at all times. This will be a critically important functionality to realize impairment-aware networking $[8,43]$. The spread-spectrum optical supervisory channel [8] used for the EON to monitor the entire spectrum at all times can be also used in 3D EON by introducing it on each OAM states. At each monitoring point, it will be necessary to use an OAM DEMUX, as the one shown above, to separate the different OAM states so that each state can be monitored independently. Performance monitoring is of particular importance in a SDM system. In fact, while different OAM states can be orthogonal in free space, there will be crosstalk once the signal will be launched and transmitted over multimode optical fibers, thus necessitating sophisticated MIMO DSP.

As we demonstrated in [8], using a real-time performance monitoring method that spans a broad and elastic spectral width, the network can dynamically and adaptively adjust the modulation format to maximize spectral efficiency while maintaining the required quality of service $(\mathrm{QoS})$ and bit error rate (BER) performance even for signals that experience time-varying physical layer impairments (QoS-aware and impairment-responsive networking). We can encode supervisory channel information at low speed (e.g., $1.25 \mathrm{~Gb} / \mathrm{s}$ ) modulation with low modulation index (e.g., $\sim 0.1$ ) on top of the data spectrum. Since the data and the supervisory channel signal follow that same path, the BER of the data is strongly correlated with that of the supervisory channel. Figure 13a shows the correlation between the measured BERs for the supervisory channel versus the data channel for three data modulation formats: BPSK, QPSK, and 8PSK. The strong 

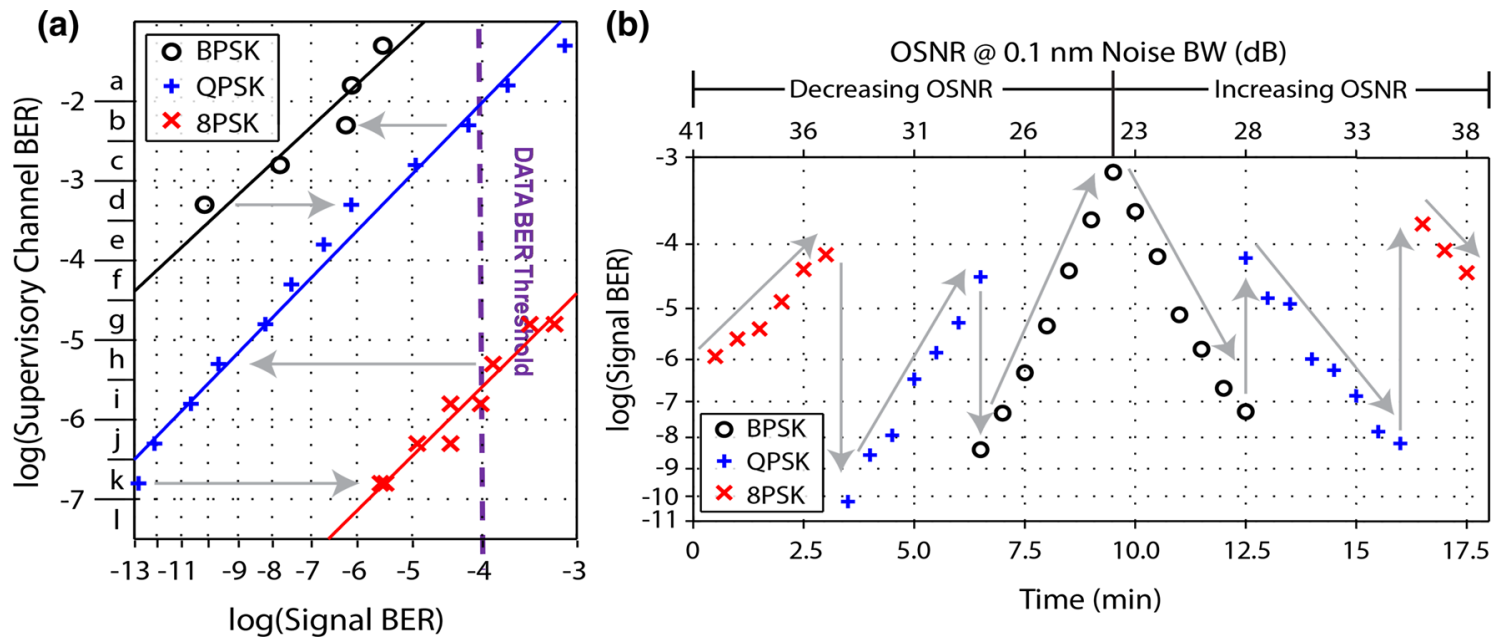

Fig. 13 a Correlation between the BER of Supervisory Channel and that of data, $\mathbf{b}$ autonomous adaptation of the QoT in the testbed shown in Fig. 14a

correlation indicates that the BER of the supervisory channel can be used to estimate the BER of the data for different modulation formats, or to choose the modulation format that maximizes the spectral bandwidth while meeting the QoS requirement (or, data $\mathrm{BER}<1 \mathrm{E}-4$ in this case). The same spread-spectrum supervisory channel can be applied to each OAM state in the OAM-based SDM system to monitor the QoT of each single OAM state $[31,44]$.

\section{Testbed integration}

Figure 14 illustrates 2D-EON testbed (temporal and spectral) at UC Davis $[45,46]$ with OAWG and OAWM EON transceivers with optical supervisory channel-based optical performance monitoring. The testbed has been upgraded to include OpenFlow-based NOX with RSSMA-based Path Computation Element (PCE). In this 3-node 2D-SDN-EON (a)

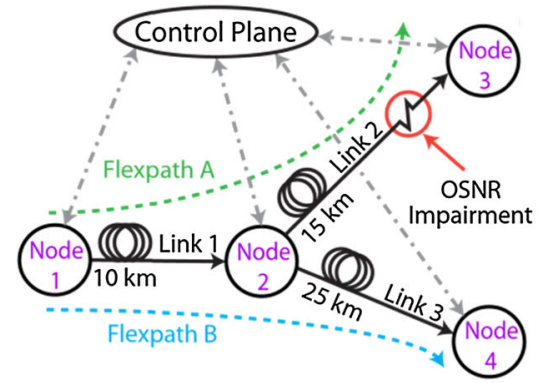

(b)

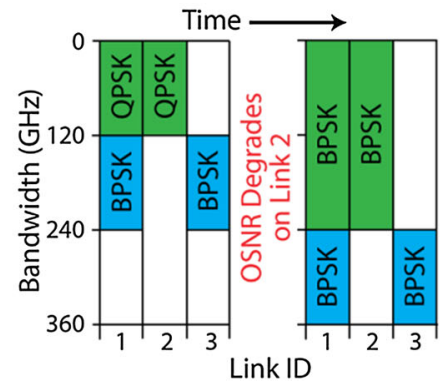

(c)

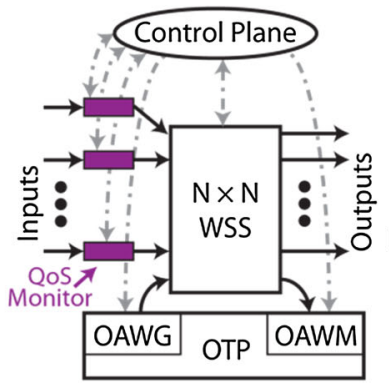

(d)

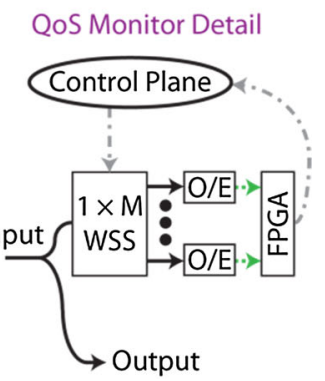

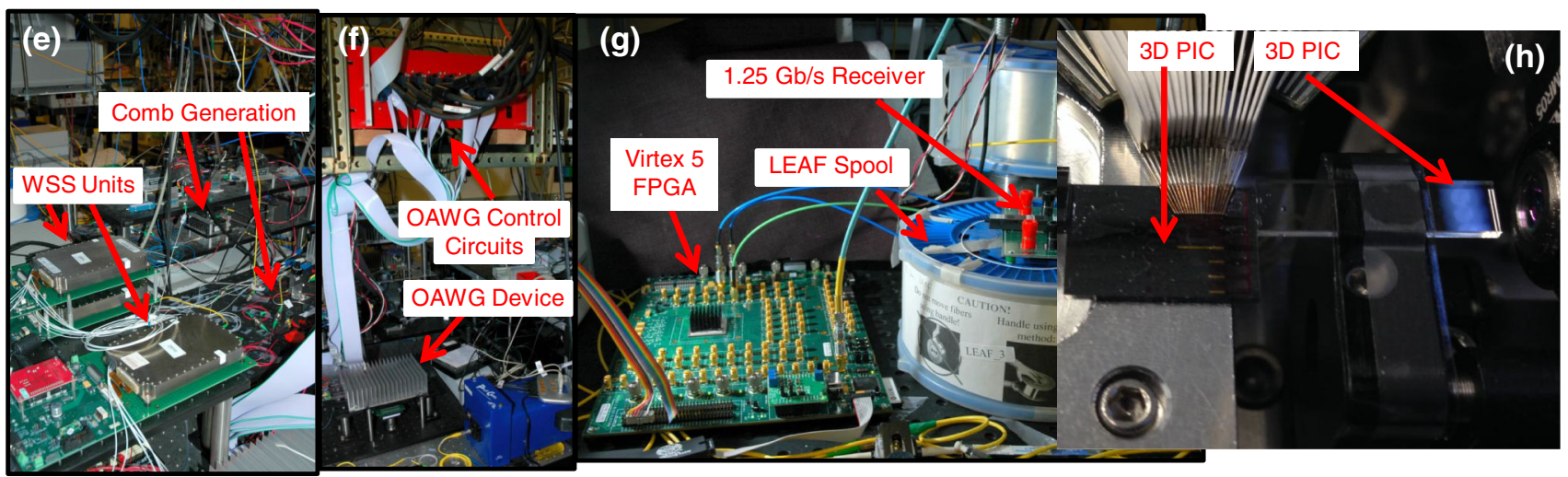

Fig. 14 a Flexible bandwidth networking scenario including timevarying OSNR on Link 2. b Spectral usage for flexpaths (corresponding to color) as OSNR degrades over time. c Flexible bandwidth wavelength cross connect (WXC) node detail. d Quality of service (QoS) monitor detail. e-h hardware testbed with OpenFlow-based NOX with PCE for $3 \mathrm{D}-\mathrm{SDN}$. (WSS wavelength-selective switch. $O A W G$ optical arbitrary waveform generation. $O A W M$ optical arbitrary waveform measurement. OTP optical transponder. FPGA field programmable gate array) 
testbed, we have shown autonomous adaptation to network impairments to maintain QoT by utilizing the observeanalyze-act cycle facilitated by optical supervisory channel. We already demonstrated transmission of multiple OAM states using the photonic integrated MUX/DEMUX device shown in Fig. 5 [31], and we have been currently working on fully integrating the OAM-based spatial dimension in the current testbed, so that it will be possible to run a full system 3D-EON demonstration with RSSMA and reconfigurable OAWG, OAWM, and OAM based on supervisory channel monitoring technique.

\section{Conclusion}

This paper presented a new approach to temporal, spectral and spatial elastic optical networking (3D-EON) exploiting, at the physical layer, optical arbitrary waveform generation and measurement techniques (OAWG and OAWM) for elasticity in the temporal and spectral domain, and orbital angular momentum technology for elasticity in the spatial domain. The control plane part of the proposed 3D-EON relies on an OpenFlow-based SDN, which is preferred to a GMPLS approach. We described the routing and spectrum, spatial mode and modulation format assignment (RSMMA) algorithm that take advantage of the additional dimension to reduce the blocking probability, and we discussed how to apply the supervisory channel monitoring technique in a 3DEON scenario. Finally, we introduced our current 2D-EON testbed and the work in progress to fully integrate the OAM dimension in the current network testbed.

Acknowledgments This work was supported by DOE under the grant DE-FC02-13ER26154, by NSF under the EECS grant 1028729, and by DARPA DSO under the grants HR0011-11-1-0005 and W911NF-12$1-0311$.

Open Access This article is distributed under the terms of the Creative Commons Attribution License which permits any use, distribution, and reproduction in any medium, provided the original author(s) and the source are credited.

\section{References}

[1] Essiambre, R., Kramer, G., Winzer, P.J., Foschini, G.J., Goebel, B.: Capacity limits of optical fiber networks. J. Lightw. Technol. 28, 662-701 (2010)

[2] Winzer, P.J.: High-spectral-efficiency optical modulation formats. J. Lightw. Technol. 30, 3824-3835 (2012)

[3] Qian, D., Huang, M.-F., Ip, E., Huang, Y.-K., Shao, Y., Hu, J., Wang, T.: $101.7-\mathrm{Tb} / \mathrm{s}(370 \times 294-\mathrm{Gb} / \mathrm{s})$ PDM-128QAM-OFDM transmission over $3 \times 55-\mathrm{km}$ SSMF using pilot-based phase noise mitigation. In: Optical Fiber Communication Conference and Exposition (OFC/NFOEC), 2011 and the National Fiber Optic Engineers Conference, pp. 1-3 (2011)
[4] Essiambre, R.J., Kramer, G., Winzer, P.J., Foschini, G.J., Goebel, B.: Capacity limits of optical fiber networks. J. Lightw. Technol. 28, 662-701 (2010)

[5] Muñoz, R., Casellas, R., Martínez, Liu, L., Tsuritani, T., Morita, I.: Experimental evaluation of efficient routing and distributed spectrum allocation algorithms for GMPLS elastic networks. Presented at the 38th European Conference and Exhibition on Optical Communications (ECOC 2012) (2012)

[6] Lowery, A.J., Wang, S., Premaratne, M.: Calculation of power limit due to fiber nonlinearity in optical OFDM systems. Opt. Express 15, 13282-13287 (Oct 2007)

[7] Yoo, S.J.B., Xin, W., Garrett, L.D., Young, J.C., Ellinas, G., Chiao, J.C., Rauch, M., Baran, J.E., Meagher, B., Leblanc, H., Chang, G.K.: Observation of prolonged power transients in a reconfigurable multiwavelength network and their suppression by gainclamping of optical amplifiers. IEEE Photonics Technol. Lett. 10, 1659-1661 (1998)

[8] Geisler, D.J., Proietti, R., Yin, Y., Scott, R.P., Cai, X., Fontaine, N.K., Paraschis, L., Gerstel, O., Yoo, S.J.B.: Experimental demonstration of flexible bandwidth networking with real-time impairment awareness. Opt. Express 19, B736-B745 (2011)

[9] Gerstel, O., Jinno, M., Lord, A., Yoo, S.J.B.: Elastic optical networking: a new dawn for the optical layer? IEEE Commun. Mag. 50, s12-s20 (2012)

[10] Jinno, M., Takara, H., Kozicki, B., Tsukishima, Y., Sone, Y., Matsuoka, S.: Spectrum-efficient and scalable elastic optical path network: architecture, benefits, and enabling technologies. IEEE Commun. Mag. 47, 66-73 (2009)

[11] Jansen, S.L., Morita, I., Schenk, T.C.W., Takeda, N., Tanaka, H.: Coherent optical 25.8-Gb/s OFDM transmission over 4160-km SSMAF. J. Lightw. Technol. 26, 6-15 (2008)

[12] Lowery, A.J., Du, L.B., Armstrong, J.: Preformance of optical OFDM in ultralong-haul WDM lightwave systems. J. Lightw. Technol. 25, 131-138 (2007)

[13] Ellis, A.D., Gunning, F.C.G.: Spectral density enhancement using coherent WDM. IEEE Photonics Technol. Lett. 17, 504-506 (2005)

[14] Bosco, G., Curri, V., Carena, A., Poggiolini, P., Forghieri, F.: On the performance of Nyquist-WDM terabit superchannels based on PM-BPSK, PM-QPSK, PM-8QAM or PM-16QAM subcarriers. J. Lightw. Technol. 29, 53-61 (2011)

[15] Scott, R.P., Fontaine, N.K., Heritage, J.P., Yoo, S.J.B.: Dynamic optical arbitrary waveform generation and measurement. Opt. Express 18, 18655-18670 (2010)

[16] Yoo, S.J.B., Scott, R.P., Geisler, D.J., Fontaine, N.K., Soares, F.M.: Terahertz information and signal processing by RF-photonics. IEEE Trans. Terahertz Sci. Technol. 2, 167-176 (2012)

[17] Geisler, D.J., Fontaine, N.K., Scott, R.P., He, T., Paraschis, L., Gerstel, O., Heritage, J.P., Yoo, S.J.B.: Bandwidth scalable, coherent transmitter based on the parallel synthesis of multiple spectral slices using optical arbitrary waveform generation. Opt. Express 19, 8242-8253 (2011)

[18] Geisler, D.J., Fontaine, N.K., He, T.T., Scott, R.P., Paraschis, L., Heritage, J.P., Yoo, S.J.B.: Modulation-format agile, reconfigurable $\mathrm{Tb} / \mathrm{s}$ transmitter based on optical arbitrary waveform generation. Opt. Express 17, 15911-15925 (2009)

[19] Geisler, D.J., Fontaine, N.K., Scott, R.P., He, T., Heritage, J.P., Yoo, S.J.B.: Single channel, $200 \mathrm{~Gb} / \mathrm{s}$, chromatic dispersion precompensated $100 \mathrm{~km}$ transmission using an optical arbitrary waveform generation based optical transmitter. In: Proceedings of the OFC 2010 (2010)

[20] Geisler, D.J., Fontaine, N.K., Scott, R.P., Yoo, S.J.B.: Demonstration of a flexible bandwidth optical transmitter/receiver system scalable to terahertz bandwidths. IEEE Photon. J. 3, 1013-1022 (2011) 
[21] Fontaine, N.K., Scott, R.P., Zhou, L., Soares, F.M., Heritage, J.P., Yoo, S.J.B.: Real-time full-field arbitrary optical waveform measurement. Nat. Photon. 4, 248-254 (2010)

[22] Ip, E., Lau, A.P.T., Barros, D.J.F., Kahn, J.M.: Coherent detection in optical fiber systems. Opt. Express 16, 753-791 (2008)

[23] Schmogrow, R., Winter, M., Meyer, M., Hillerkuss, D., Wolf, S., Baeuerle, B., Ludwig, A., Nebendahl, B., Ben-Ezra, S., Meyer, J., Dreschmann, M., Huebner, M., Becker, J., Koos, C., Freude, W., Leuthold, J.: Real-time Nyquist pulse generation beyond 100 Gbit/s and its relation to OFDM. Opt. Express 20, 317-337break (2012)

[24] Allen, L., Beijersbergen, M.W., Spreeuw, R.J.C., Woerdman, J.P.: Orbital angular momentum of light and the transformation of Laguerre-Gaussian laser modes. Phys. Rev. A 45, 8185-8189 (1992)

[25] Bozinovic, N., Yue, Y., Ren, Y., Tur, M., Kristensen, P., Huang, H., Willner, A.E., Ramachandran, S.: Terabit-scale orbital angular momentum mode division multiplexing in fibers. Science 340, 1545-1548 (2013)

[26] Su, T., Scott, R.P., Djordjevic, S.S., Fontaine, N.K., Geisler, D.J., Cai, X., Yoo, S.J.B.: Demonstration of free space coherent optical communication using integrated silicon photonic orbital angular momentum devices. Opt. Express 20, 9396-9402 (2012)

[27] Guan, B. Scott, R.P., Fontaine, N.K., Su, T., Ferrari, C., Cappuzzo, M., Klemens, F., Keller, B., Earnshaw, M., Yoo, S.J.B.: Integrated optical orbital angular momentum multiplexing device using 3-D waveguides and a silica PLC. In: CLEO: 2013, San Jose, CA, p. CTu1L.4 (2013)

[28] Scott, R.P., Guan, B., Qin, C., Fontaine, Su, N.K.T., Ferrari, C., Cappuzzo, M., Klemens, F., Keller, B., Earnshaw, M., Yoo, S.J.B.: Free-space coherent optical communication demonstration using a 3D photonic integrated circuit device for orbital angular momentum multiplexing/demultiplexing. In: European Conference and Exhibition on Optical Communication (ECOC), p. Tu.3.B.2 (2013)

[29] Macdonald, J.R., Thomson, R.R., Beecher, S.J., Psaila, N.D., Bookey, H.T., Kar, A.K.: Ultrafast laser inscription of nearinfrared waveguides in polycrystalline ZnSe. Opt. Lett. 35, 40364038 (2010)

[30] Thomson, R.R., Bookey, H.T., Psaila, N.D., Fender, A., Campbell, S., MacPherson, W.N., Barton, J.S., Reid, D.T., Kar, A.K.: Ultrafast-laser inscription of a three dimensional fan-out device for multicore fiber coupling applications. Opt. Express 15, 1169111697 (2007)

[31] Guan, B., Scott, R.P., Qin, C., Fontaine, N.K., Su, T., Ferrari, C., Cappuzzo, M., Klemens, F., Keller, B., Earnshaw, M., Yoo, S.J.B.: Free-space coherent optical communication with orbital angular, momentum multiplexing/demultiplexing using a hybrid 3D photonic integrated circuit. Opt. Express 22, 145-156 (2014)

[32] Patel, A.N., Ji, P.N., Jue, J.P., Wang, T.: Routing, wavelength assignment, and spectrum allocation in transparent flexible optical WDM (FWDM) networks. In: Photonics in Switching, p. PDPWG1 (2010)

[33] Christodoulopoulos, K., Tomkos, I., Varvarigos, E.A.: Elastic bandwidth allocation in flexible OFDM-based optical networks. J. Lightw. Technol. 29, 1354-1366 (2011)

[34] Jinno, M., Kozicki, B., Takara, H., Watanabe, A., Sone, Y., Tanaka, T., Hirano, A.: Distance-adaptive spectrum resource allocation in spectrum-sliced elastic optical path network [topics in optical communications]. IEEE Commun. Mag. 48, 138-145 (2010)

[35] Liu, L., Yin, Y., Xia, M., Shirazipour, M., Zhu, Z., Proietti, R., Xu, Q., Dahlfort, S., Yoo, S. B.: Software-defined fragmentationaware elastic optical networks enabled by OpenFlow. In: 39th European Conference and Exhibition on Optical Communication (ECOC 2013), pp. 1-3 (2013)
[36] Wen, K., Cai, X.R., Yin, Y.W., Geisler, D.J., Proietti, R., Scott, R.P., Fontaine, N.K., Yoo, S.J.B.: Adaptive spectrum control and management in elastic optical networks. IEEE J. Sel. Areas Commun. 31, 39-48 (2013)

[37] Yin, Y., Zhang, H., Zhang, M., Xia, M., Zhu, Z., Dahlfort, S., Yoo, S.J.B.: Spectral and spatial 2D fragmentation-aware routing and spectrum assignment algorithms in elastic optical networks [invited]. J. Opt. Commun. Netw. 5, A100-A106 (2013)

[38] Qin, C., Guan, B., Scott, R., Proietti, R., Fontaine, N., Su, T., Ferarri, C., Capuzzo, M., Clemens, F., Keller, B., Earnshaw, M., Yoo, S.J.B.: Demonstration of Orbital Angular Momentum State Conversion Using Two Hybrid 3D Photonic Integrated Circuits Technical Digest Optical Fiber Communication Conference, vol. Paper Th4A.1 (2014)

[39] Proietti, R., Qin, C., Guan, B., Yin, Y., Scott, R.P., Yu, R., Yoo, S.J.B.: Rapid and complete hitless defragmentation method using a coherent RX LO with fast wavelength tracking in elastic optical networks. Opt. Express 20, 26958-26968 (2012)

[40] Liu, L., Tsuritani, T., Morita, I., Guo, H., Wu, J.: Experimental validation and performance evaluation of OpenFlow-based wavelength path control in transparent optical networks. Opt. Express 19, 26578-26593 (2011)

[41] Das, S., Parulkar, G., McKeown, N.: Why OpenFlow/SDN can succeed where GMPLS failed. Presented at the European Conference and Exhibition on Optical Communications (ECOC 2012), Amsterdam, Netherlands (2012)

[42] Liu, L., Tsuritani, T., Morita, I., Casellas, R., Martínez, R., Muñoz, R.: Control plane techniques for elastic optical networks: GMPLS/PCE vs OpenFlow. In: International Workshop on Flexible Optical Networks, IEEE Global Communications Conference (GLOBECOM), pp. 352-357, Anaheim, CA, USA (2012)

[43] Cai, X.R., Wen, K., Proietti, R., Yin, Y.W., Geisler, D.J., Scott, R.P., Qin, C., Paraschis, L., Gerstel, O., Yoo, S.J.B.: Experimental demonstration of adaptive combinational QoT degradation restoration in elastic optical networks. J. Lightw. Technol. 31, 664-671 (2013)

[44] Ren, Y., Zhang, Y., Yue, Y., Bozinovic, N., Xie, G., Huang, H., Tur, M., Kristensen, P., Djordjevic, I.B., Ramachandran, S., Willner, A.E.: Efficient crosstalk mitigation of OAM based 400-Gbit/s QPSK data transmission in 1.1-km vortex fiber by using softdecision LDPC codes. In: CLEO, p. CM2G.5, San Jose, CA (2013)

[45] Wen, K., Yin, Y., Geisler, D., Chang, S., Yoo, S.J.B.: Dynamic on-demand lightpath provisioning using spectral defragmentation in flexible bandwidth networks. In: ECOC 2011, p. Mo.2.K.4, Geneva, Switzerland (2011)

[46] David, R.P., Geisler, J., Yin, Y., Scott, R.P., Cai, X., Fontaine, N.K., Paraschis, L., Gerstel, O., Yoo, S.J.B.: The first testbed demonstration of a flexible bandwidth network with a real-time adaptive control plane. In: ECOC 2011, p. Th.13.K.2, Geneva, Switzerland (2011)

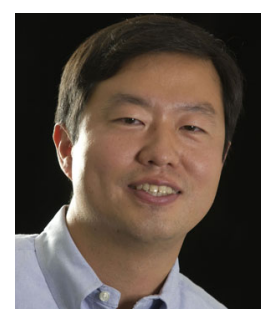

S. J. Ben Yoo (S'82-M'84-SM'97-F'07) received the B.S. degree in electrical engineering with distinction, the M.S. degree in electrical engineering, and the Ph.D. degree in electrical engineering with a minor in physics, all from Stanford University, Stanford, CA, in 1984, 1986, and 1991, respectively. He currently serves as Professor of Electrical Engineering at University of California at Davis (UC Davis). His research at UC Davis includes high-performance all-optical devices, systems, and networking technologies for future computing and communications. Prior to joining UC Davis in 1999, he was a senior research scientist at Bellcore, leading technical efforts in optical networking research and 
systems integration. He participated ATD/MONET testbed integration and a number of standardization activities including GR-2918-CORE, GR-2918-ILR, GR-1377-CORE, and GR-1377-ILR on dense WDM and OC-192 systems. He is a Fellow of the Optical Society of America (OSA), a Fellow of the IEEE, a Fellow of NIAC (NASA), and is a recipient of the DARPA Award for Sustained Excellence (1997), the Bellcore CEO Award (1998), the Outstanding Mid-Career Research Award (UC Davis, 2004), and the Outstanding Senior Research Award (UC Davis, 2011).

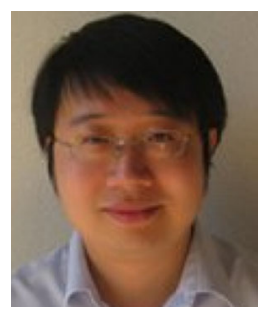

Lei Liu received B.E. and Ph.D. degrees from Beijing University of Posts and Telecommunications (BUPT), Beijing, China, in 2004 and 2009 respectively. From 2009 to 2012, he was a research engineer at KDDI R\&D Laboratories Inc., Japan, where he was engaged in research \& develop on intelligent optical networks and their control and management technologies. He is now with the University of California at Davis, USA. His main research interests include the wavelength switched optical networks, elastic optical networks, network control and management techniques (such as MPLS/GMPLS, PCE, OpenFlow), software-defined networking (SDN), network architectures and grid/cloud computing. He has coauthored more than 100 peer-reviewed papers related to the previously mentioned research topics in international journals and conferences, and he is the first author of more than 50 of them. He has presented several invited talks in the international conferences, including some prestigious ones such as ECOC'11, ECOC'12 and Globecom'12.

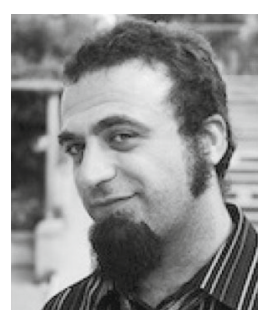

Roberto Proietti is a Project Scientist with the Next Generation Networking Systems Laboratory, University of California, Davis. $\mathrm{He}$ received the M.S. degree in Telecommunications Engineering from University of Pisa, Italy, in 2004 and the $\mathrm{PhD}$ in Electrical Engineering from Scuola Superiore Sant' Anna, Pisa, Italy in 2009. His research interests include optical switching technologies and architectures for supercomputing and data center applications, high-spectrum efficiency coherent transmission systems and elastic optical networking, access optical networks and radio over fiber.

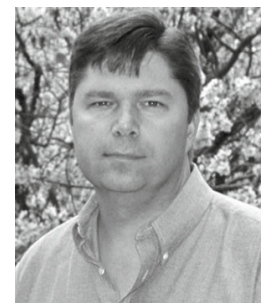

Ryan P. Scott (S'93-M'03) received the B.S. degree in laser electrooptics technology from the Oregon Institute of Technology, Klamath Falls, in 1991, the M.S. degree in electrical engineering from the University of California, Los Angeles (UCLA), in 1995 and the Ph.D. degree in electrical and computer engineering from the University of California, Davis (UC Davis) in 2009. He is currently an Asst. Project Scientist at UC Davis. His present research interests include optical arbitrary waveform generation and measurement, optical comb generation, spatial division multiplexing, non-traditional imaging, and femtosecond laser writing of waveguides in bulk dielectric materials. 